\title{
A Model for the Formation of the Milky Way
}

\author{
Markus Samland ${ }^{1,2}$ \\ ${ }^{1}$ Astronomisches Institut der Universität Basel, CH 4102 Binningen, Switzerland \\ ${ }^{2}$ E-mail: samland@astro.unibas.ch
}

Received 2003 October 16, accepted 2004 January 19

\begin{abstract}
A new chemodynamical model for the formation and evolution of a Milky Way type galaxy is introduced. In this scenario, the galaxy forms inside a slowly growing dark matter halo in a $\Lambda$ CDM cosmology. In contrast to the simple merger and collapse scenarios, the galactic mass grows continuously over a Hubble time. The whole formation scenario is simulated with a three-dimensional chemodynamical code. Within this model it is possible to follow the evolution of the galactic substructure in detail. The structure of the galactic components - halo, bulge, and disk - and the kinematical and chemical signatures of the stellar populations in the model are in excellent agreement with data from the Milky Way. The present model provides a detailed formation scenario for the Milky Way Galaxy and it yields new information about its kinematical and chemical history. The model predicts that even galaxies like the Milky Way show phases with supernova-driven galactic winds. However, with a mass loss of the order of only a few per cent of the total baryonic mass, these galaxies are in all probability not the main contributors to the enrichment of the intergalactic medium.
\end{abstract}

Keywords: Galaxy: kinematics and dynamics — ISM: kinematics and dynamics — Galaxy: evolution Galaxy: stellar content — Galaxy: formation

\section{Introduction}

During the last decade large efforts were made to consolidate a standard cosmological model. In the presently favoured $\Lambda \mathrm{CDM}$ cosmology the universe has a hierarchical structure in which first the small and later the more massive dark halos form. These massive dark halos are the hosts of galaxies like the Milky Way. Although the assembly of the dark matter seems to be understood in general, galaxy formation inside the dark halos is not well established. Galaxy formation is a complex network of physical processes controlled by the total mass and angular momentum, the halo formation history, and the environment. Processes like star formation, the star-gas interactions, or the interplay between the different phases of the interstellar medium (ISM) have a determining influence on the final shape and structure of a galaxy as well.

In former times the purity of gold and silver was tested by the streak left upon a touchstone that had been rubbed by the metal. The streaks are compared with streaks made by metals of known purity. In the field of galactic evolution, these 'streaks on the touchstone' are the substructure of a galaxy (e.g. thick and thin disks, bulge-to-disk ratios, stellar streams, gas-star fractions, metallicity gradients, or density profiles), the distribution of stellar orbits, and the chemical abundances of stars. If we want to interpret these signatures it is necessary to have detailed models of the galaxy formation process which provide the comparative galactic 'streaks'.

The chemical and kinematical data of Galactic halo stars have been one of the starting points for different scenarios for the formation and evolution of galaxies. It is interesting to note that both the collapse model of Eggen et al. (1962) and the merger model of Searle \& Zinn (1978) are based on data of Galactic halo stars. This has two reasons - firstly, it is difficult to get reliable data for halo stars and, secondly, for both scenarios there had been no detailed model which really showed the characteristic kinematical and chemical signatures. Samland et al. (1997) showed that early homogeneous collapse models are oversimplified, because feedback processes and the physics of the ISM influence the protogalactic collapse. This illustrates the importance of numerical models in interpreting the kinematical and chemical data of stellar populations. The simple scenarios are also ruled out by the new data, e.g. the proper motion catalogue of Galactic stars from Chiba \& Beers (2000).

In principle, a numerical model is only a first-order approximation to a real galaxy. But even with the numerical models available at the moment, we can learn much about the important processes, and the comparison with observations leads to further improvements of the models. That is also one reason why a formation model of the Milky Way is so important - it is the best observed galaxy and therefore the test case for any galactic model. When we know how the Milky Way has formed, it will be only a small step to understand the formation process of other galaxies.

\section{The Chemodynamical Model}

The present chemodynamical model is described in Samland \& Gerhard (2003) and further references are given there. In summary, it is a three-dimensional hybrid 

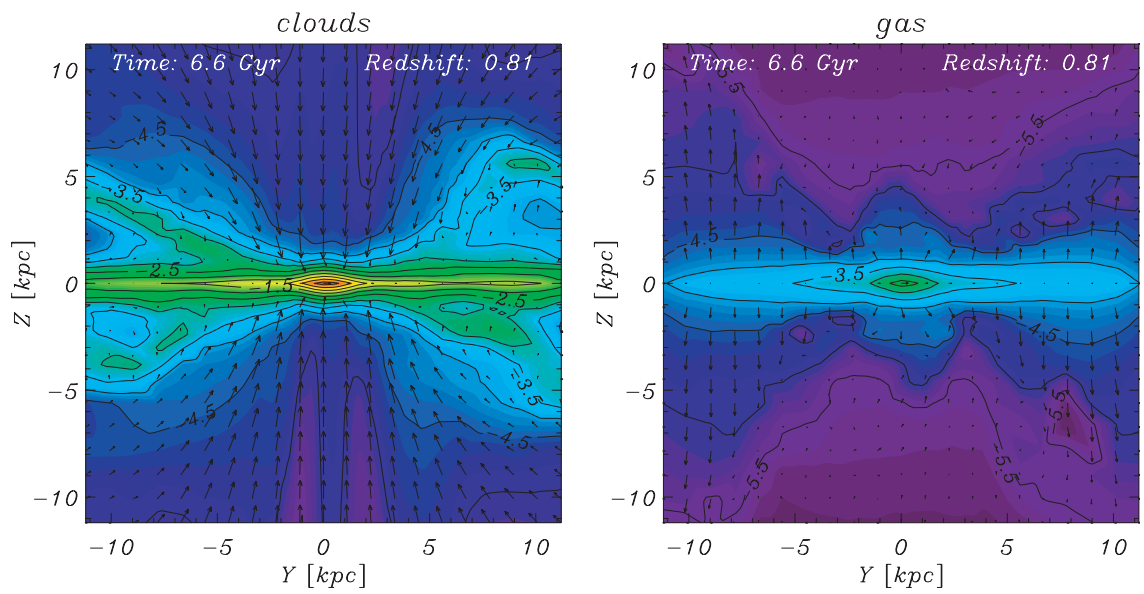

Figure 1 The two panels show the density and the velocity fields of the ISM phases at redshift 0.8 . Note the different flow patterns of the cloud and the gas phases. The density decreases from red to green to blue.

code consisting of a hydrodynamical grid code for the ISM phases and a particle-mesh code for the stars, stellar remnants, and dark matter. The stars and the multiphase ISM are connected by an interaction network describing star formation, stellar evolution, star death, heating and cooling, dissipation, and ISM mixing processes. Dark and baryonic matter are coupled only by one force: gravitation. The present model differs from the published model of Samland \& Gerhard (2003) in one point. The dark halo formation history is no longer extracted from the VIRGO-GIF database (see Kauffmann et al. 1999); instead I used the halo formation history given by Wechsler et al. (2002) together with the angular momentum distribution published by Bullock et al. (2001). That is why the halo formation history can be described on the basis of four parameters: the total mass $M$ (baryonic and dark), the halo formation redshift $z_{\mathrm{F}}$, the spin parameter $\lambda$ (total amount of angular momentum), and the shape parameter $\mu$ (spatial distribution of the angular momentum). To simulate a Milky Way type galaxy I chose the following values: $M=6 \times 10^{11} \mathrm{M}_{\odot}, z_{\mathrm{F}}=4.0, \lambda=0.05$, and $\mu=1.25$. This model is closely related to the accretion model described in Samland \& Gerhard (2003), except the total mass which, in the present case, is a factor of three lower.

\section{The Importance of the Multiphase Interstellar Medium}

There are two basic differences between the present and other chemodynamical models. Firstly, a grid-based hydrodynamical code is used to simulate the dynamics of the different phases of the ISM. The advantage is the well-balanced spatial resolution and, more importantly, the artificial viscosity can be reduced to small values, ensuring the conservation of angular momentum. The second difference concerns the description of the ISM. In the present case, the three-phase model of McKee \& Ostriker (1977) is adopted. However, since the dynamics of the cold cloud cores and the warm cloud envelopes are coupled, only two distinct dynamical phases exist. Most of the mass in the ISM is in the star-forming cloudy phase which has low specific energy densities. In contrast to this, the gas phase is hot and pressure-dominated. The energy in this second phase is provided by stellar winds of massive stars and by supernovae explosions. As an example, Figure 1 shows the density and the velocity fields of these two components at a redshift $z=0.81$ in a plane perpendicular to the galactic disk. It is obvious that at the same time the galaxy shows infall of clouds and outflow of hot and mostly metal-rich gas. These outflows are important, because they enrich the galactic halo and, in some cases, even the intergalactic medium.

Using a one-phase instead of a two-phase medium in a galactic model causes large differences in the galaxy formation process. With the one-phase medium the galactic disks are more concentrated (smaller scale lengths), the bulge masses are larger, and the star formation rates peak at earlier times (with a time shift of 1-2 Gyr). The models with the two-phase medium show stronger mass circulations which show up in higher temperatures and metallicities of the halo gas and a higher fraction of galactic mass loss. More results about the importance of the multiphase treatment of the ISM will be published in a forthcoming paper.

\section{The Galactic Evolution in Five Steps}

The formation of a massive disk galaxy shows different evolutionary phases. In this section I give a short outline of the formation process as found from the chemodynamical evolution model.

- From redshift $z=10$ to redshift $z=2$.

At high redshifts $z>2$ the mass of the dark halo increases dramatically, and a massive protogalactic cloud begins to form. This cloud is not stable, because dissipation and cooling processes are very efficient in reducing the internal pressure. As a result the protogalactic cloud begins to collapse. During this collapse, the inner stellar halo and a protobulge form. The most striking circumstance about this phase is the fact that the star formation is located in separated clumps (see 
also Immeli et al. 2004) and that the supernovae drive a galactic wind.

- From redshift $z=2$ to redshift $z=1$.

During the collapse, the star formation rate increases and in consequence the stellar feedback becomes more efficient. At a redshift of $z=2$, the collapse turns into a quasi-static contraction. In the following period, the flow of cold and warm gas into the inner galaxy decreases and the star formation rate begins to drop. At the end of this evolution step, the bulge and most of the thick disk have formed. The feedback by supernovae gives rise to strong distortions in the early thick disk and it drives flows of metal-rich gas from the bulge into the disk.

- From redshift $z=1$ to redshift $z=0.5$.

Between redshift $z=1$ to $z=0.5$ star formation moves more and more from the inner galaxy into the galactic plane, where the gas is already pre-enriched. A thin stellar disk forms. In this phase the most metal-rich stars of the galaxy are born in the bulge and inner disk. Later, the metallicity decreases again slightly, due to the infall of low-metallicity gas and, more importantly, the stellar mass return from old stars.

- From redshift $z=0.5$ to redshift $z=0.2$.

At redshift $z=0.5$ the galaxy has achieved its final shape and a 'quiet evolution phase' begins. The disk still grows radially, however very slowly, and, in the present case, the star formation levels off at a rate of $6 \mathrm{M}_{\odot} \mathrm{yr}^{-1}$, concentrated in the galactic plane. At galactocentric radii of $15-20 \mathrm{kpc}$, the gaseous disk shows a warp, which, in the stellar disk, is only slightly visible. The cause of this warp is still not known, but the feedback from stars in the outer galactic disk seems to play a role.

- From redshift $z=0.2$ to redshift $z=0.0$.

This last phase in the evolution of the model galaxy is characterised by the stellar mass return from low mass stars and the infall of pre-enriched intergalactic gas. As the dark halo grows it can capture gas expelled at early stages. This gas, enriched up to 0.25 of the solar metallicity, can fall back into the galactic disk and together with the gas returned from the old stellar populations can trigger new star formation events. The average metallicity of this returned gas ranges from 0.1 to 1.0 times the solar metallicity, however with solar $[\alpha / \mathrm{Fe}]$ ratios.

\section{Selected Results}

In the following I summarise a few basic results of the present model. These results will be discussed in more detail in forthcoming publications. The oldest galactic component in the model is the stellar halo, which, for the most part, forms during the initial collapse at redshifts larger than $z=2$. The halo stars have on average low metallicities $([\mathrm{Fe} / \mathrm{H}]<-1)$ but the abundance scatter from star to star is large (see Figure 2). The halo forms from inside out with a clear radial age gradient, but no sign of a metallicity gradient (Figure 2). This is in agreement

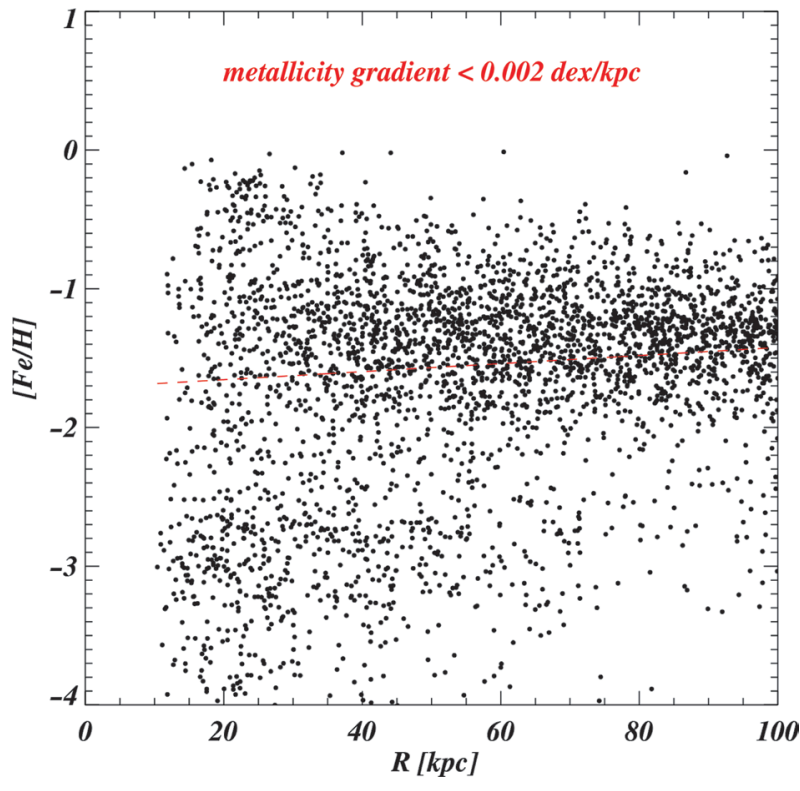

Figure 2 Radial $[\mathrm{Fe} / \mathrm{H}]$ distribution of halo stars in the model. The metallicity gradient $\left(0.002 \mathrm{dex} \mathrm{kpc}^{-1}\right)$ is very shallow and the scatter from star to star is large.

with the halo formation model of Bekki \& Chiba (2001) and the findings of Chiba \& Beers (2000), who analysed the kinematics and chemical composition of Galactic halo stars. In the present model, the rare 'younger' halo stars have unusual chemical compositions. While the metallicities are as low as $[\mathrm{Fe} / \mathrm{H}]=-2$, the $[\alpha / \mathrm{Fe}]$ ratios are nearly solar. Chemically these stars are very similar to the stars seen in some of the local dwarf spheroidal galaxies (Shetrone et al. 2003). The model of Raiteri et al. (1996) also shows such a stellar population with $[\mathrm{Fe} / \mathrm{H}]<-1.0$ and low $[\alpha / \mathrm{Fe}]$ ratios.

Looking at the relations between the kinematical and chemical properties of the stellar populations, I find the following trends: stars with higher metallicities have, on average, lower $[\alpha / \mathrm{Fe}]$ ratios, less eccentric orbits, and a higher rotation speed around the galactic centre (see Figure 18 in Samland \& Gerhard 2003). However, the scatter in these quantities is large and there are stars with low metallicities moving on circular orbits and also high metallicity stars with radial orbits. The average age-metallicity relation of the model stars is very similar to the one found for Galactic stars (e.g. Buser 2000). However, it may be inappropriate to speak of an age-metallicity relation, because at any given age the stellar metallicities show a scatter of a factor of ten.

A last result I want to address is the galactic mass loss rates measured with the chemodynamical models. In the present case the mass loss is only $2.4 \%$ of the total baryonic mass. However, for other massive galaxies, the chemodynamical simulations predict mass losses up to $10 \%$ of the baryonic mass. As expected, the mass loss decreases with increasing mass of a galaxy, and it increases with angular momentum. For dwarf galaxies, the model predicts mass losses up to $80 \%$ of the baryonic 


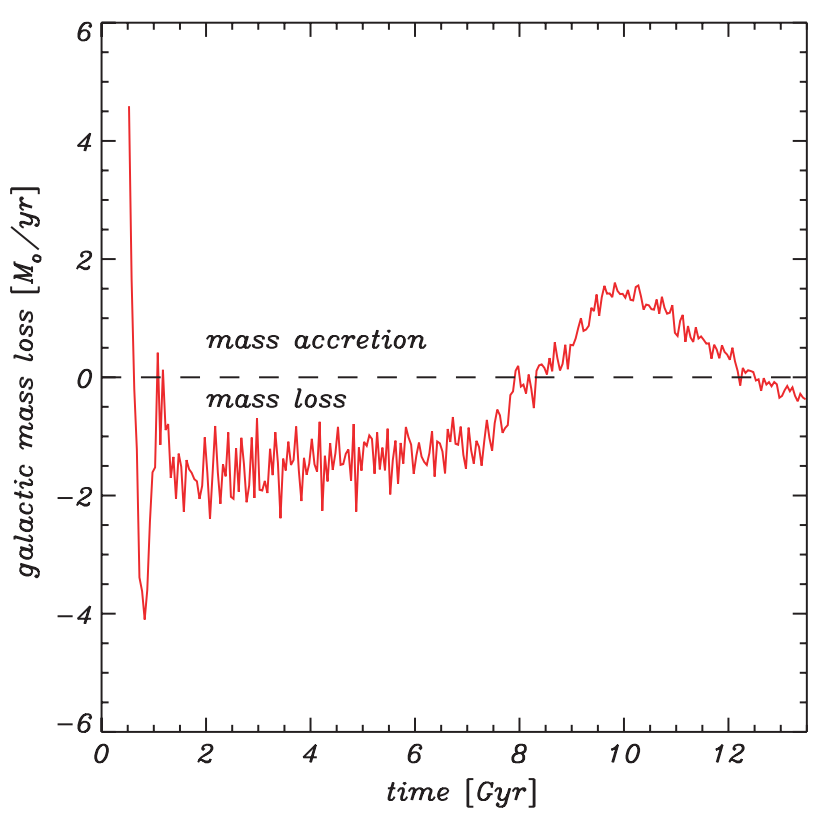

Figure 3 Mass loss by supernova-driven winds occur during the early galactic evolution. Later, a part of this material can be captured again by the galaxy.

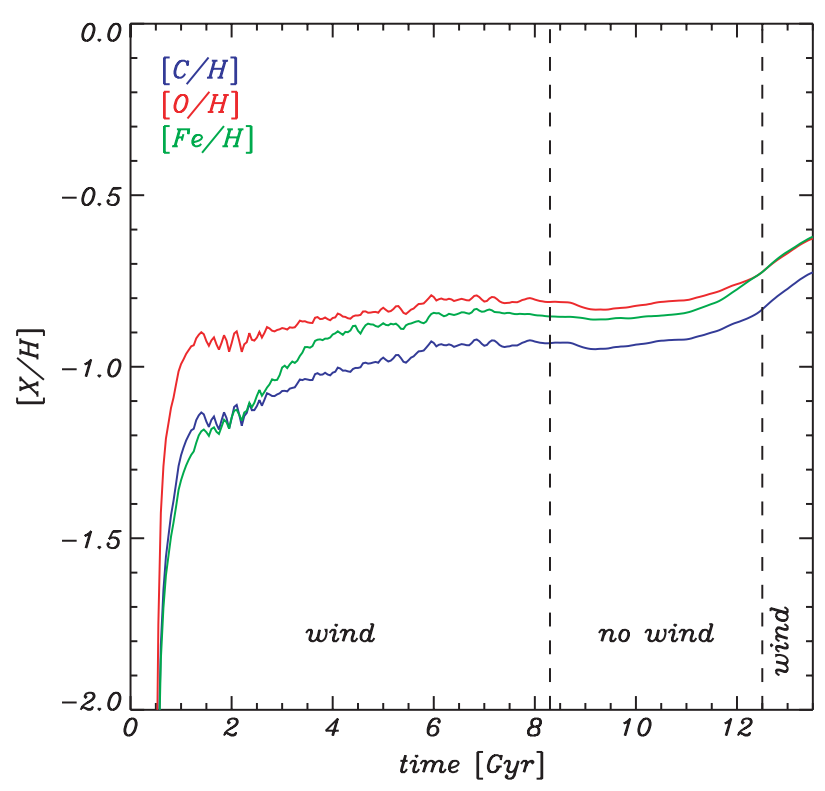

Figure 4 The evolution of the chemical abundances of carbon, oxygen, and iron in the supernova-driven galactic wind.

matter. The mass loss manifests itself as a supernovadriven galactic wind, with a changing intensity during the galactic evolution. In Figure 3 I have plotted the net infall and outflow rates, neglecting the slow accretion of matter given by the cosmological background model. At redshifts $z>0.5$, the simulations show a supernova-driven mass loss of the order of $1-2 \mathrm{M}_{\odot} \mathrm{yr}^{-1} .1$ At high redshifts the mass loss can be greater, and at low redshifts

\footnotetext{
${ }^{1}$ The given mass loss is uncertain by a factor of two to three, taking into account the model uncertainties of both, the supernova feedback, and the mixing processes in the ISM.
}

a part of the expelled gas is captured again by the galaxy. The typical metallicity of the expelled gas is in the range $-1<[\mathrm{Fe} / \mathrm{H}]<-0.4$ (Figure 4). Only at redshifts $z>2.3$ is the chemical composition of the outflowing gas dominated by Type II supernovae. At $z=1.6$, the $[\alpha / \mathrm{Fe}]$ ratio in the wind is already solar.

\section{Conclusion}

The presented model of a galaxy forming in a slowly growing dark matter halo gives a consistent description for the formation of a Milky Way type galaxy. It is based on the cosmological models of structure formation and it is a new formation scenario differing from the classical collapse (Eggen et al. 1962) and merger scenarios (Searle \& Zinn 1978). I call this scenario a galactic accretion model because the galaxy mass grows continuously without any major mergers. In this scenario, there is also a collapse phase, but this collapse differs significantly from the monolithic collapse scenario proposed by Eggen et al. (1962). A basic finding is that the galaxy forms slowly from inside out and from halo to disk. The time to build the galactic components (halo, bulge, and disk) depends mainly on the mass accretion history of the dark halo, the angular momentum of the accreted matter, and the stellar feedback processes. More information about this formation scenario can be found in Samland \& Gerhard (2003). The kinematical and chemical signatures of the stellar populations in the model are in excellent agreement with data from the Milky Way (e.g. Chiba \& Beers 2000). The present model describes a self-consistent Milky Way formation scenario which matches the chemical and kinematical data of Galactic stellar populations.

\section{Acknowledgements}

I thank the organisers of the 5th Workshop on Galactic Chemodynamics for the invitation. The simulations were performed at the Swiss Centre for Scientific Computing (CSCS) in Manno.

\section{References}

Bekki, K., \& Chiba, M. 2001, ApJ, 558, 666

Bullock, J. S., Dekel, A., Kolatt, T. S., Kravtsov, A. V., Klypin, A. A., Porciani, C., \& Primack, J. R. 2001, ApJ, 555, 240

Buser, R. 2000, Science, 287, 69

Chiba, M., \& Beers, T. C. 2000, AJ, 119, 2843

Eggen, O. J., Lynden-Bell, D., \& Sandage, A. R. 1962, ApJ, 136,748

Immeli, A., Samland, M., Gerhard, O. E., \& Westera, P. 2004, A\&A, 413, 547

Kauffmann, G., Colberg, J. M., Diaferio, A., \& White, S. D. M. 1999, MNRAS, 303, 188

McKee, C. F., \& Ostriker, J. P. 1977, ApJ, 218, 148

Raiteri, C. M., Villata, M., \& Navarro, J. F. 1996, A\&A, 315

Samland, M. \& Gerhard, O. E. 2003, A\&A, 399, 961

Samland, M., Hensler, G., \& Theis, C. P. 1997, ApJ, 476, 544

Searle, L., \& Zinn, R. 1978, ApJ, 225, 357

Shetrone, M., Venn, K. A., Tolstoy, E., Primas, F., Hill, V., \& Kaufer, A. 2003, AJ, 125, 684

Wechsler, R. H., Bullock, J. S., Primack, J. R., Kravtsov, A. V., \& Dekel, A. 2002, ApJ, 568, 52 